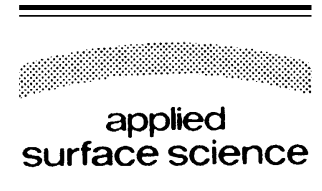

Applied Surface Science 238 (2004) 151-154

www.elsevier.com/locate/apsusc

\title{
Electron inelastic mean free paths and surface excitation parameters for GaAs
}

\author{
C.M. Kwei", Y.C. Li \\ Department of Electronics Engineering, National Chiao Tung University, Hsinchu 300, Taiwan
}

Available online 27 July 2004

\begin{abstract}
Surface excitation parameters and inelastic mean free paths of electrons are of importance in the analyses of surface sensitive electron spectroscopies. When probe electrons are near the surface of a solid or the interface of an overlayer system, electron inelastic mean free paths become depth-dependent. These mean free paths and surface excitation parameters were calculated for electrons crossing the surface of GaAs. Calculations were performed for both incident and outgoing electrons by the use of a dielectric response theory. Applications were made to estimate the elastic backscattering intensity of electrons at different emission angles using the Monte Carlo simulations. Good agreement was found between calculated results and experimental data on the ratio of the elastic reflection coefficient for a GaAs sample relative to a $\mathrm{Ni}$ reference. Such a ratio was used to determine the effective electron inelastic mean free paths in GaAs by employing the surface excitation parameter obtained from Monte Carlo simulations.
\end{abstract}

(C) 2004 Elsevier B.V. All rights reserved.

PACS: 72.10.-d; 73.20.Mf; 78.20.Bh

Keywords: Surface excitation parameter; Inelastic mean free path; Monte Carlo simulation

\section{Introduction}

Electron inelastic mean free path (IMFP) plays an important role in the quantitative analysis of surface sensitive electron spectroscopies. The IMFP can be extracted, for instance, from reflected elastic peak electron spectra [1]. Corrections should be made in such extractions to account for the contribution from surface excitations [2-4]. Alternatively, the IMFP can be calculated by considering all relevant interactions including volume and surface excitations based on the dielectric response theory. The surface excitations are

\footnotetext{
* Corresponding author. Tel.: +886 3 5712121x54136; fax: +88635727300 .

E-mail address: cmkwei@mail.nctu.edu.tw (C.M. Kwei).
}

generated by electrons extending to both sides of the surface to a distance in the order of angstroms [5]. These excitations, characterized by the surface excitation parameter (SEP), lead to a depth-dependent electron IMFP inside the solid [5]. They also cause a significant influence on the elastic backscattering intensity [6].

In the present work, the depth-dependent IMFPs and SEPs of electrons incident into or escaping from GaAs were calculated using the extended Drude dielectric function $[7,8]$. Calculated IMFPs and SEPs were applied to determine the electron elastic backscattering intensity by Monte Carlo (MC) simulations [9,10]. An effective IMFP was subsequently extracted from this intensity. Such a procedure was similar to the method used in the experimental extraction of IMFPs $[11,12]$. 


\section{Electron inelastic mean free paths and surface excitation parameters}

Considering a semi-infinite solid $(z>0)$ with the dielectric function $\varepsilon(\rightarrow q, \omega)$, the inverse IMFP at depth $z$ is given by $[5,6]$

$$
\begin{aligned}
& \left.\lambda_{i}^{-1}(E, z)\right|_{v \rightarrow s} \\
& =\frac{2}{\pi v} \int_{0}^{E} \mathrm{~d} \omega \int_{Q_{-}}^{Q_{+}} \mathrm{d} Q \frac{v Q}{\omega^{2}+(v Q)^{2}}\left\{2 \eta \mathrm{e}^{-2 Q|z|} \Theta(-z)\right. \\
& \quad+\left\{2 \eta \mathrm{e}^{-Q|z|}\left[2 \cos \left(\frac{\omega z}{v}\right)-\mathrm{e}^{-Q|z|}\right]\right. \\
& \left.\left.\quad+\xi\left[1+\mathrm{e}^{-2 Q|z|}-2 \mathrm{e}^{-Q|z|} \cos \frac{\omega z}{v}\right]\right\} \Theta(z)\right\}
\end{aligned}
$$

for normally incident (from vacuum to solid) electrons of speed $v$ and energy $E=v^{2} / 2$, and

$$
\begin{aligned}
\left.\lambda_{i}^{-1}(E, z)\right|_{s \rightarrow v} & \\
= & \frac{2}{\pi v} \int_{0}^{E} \mathrm{~d} \omega \int_{Q_{-}}^{Q_{+}} \mathrm{d} Q \frac{v Q}{\omega^{2}+(v Q)^{2}} \\
& \times\left\{2 \eta \mathrm{e}^{-Q|z|}\left[2 \cos \left(\frac{\omega z}{v}\right)-\mathrm{e}^{-Q|z|}\right] \Theta(-z)\right. \\
& \left.+\left[2 \eta \mathrm{e}^{-2 Q|z|}+\xi\left(1-e^{-2 Q|z|}\right)\right] \Theta(z)\right\}
\end{aligned}
$$

for normally escaping (from solid to vacuum) electrons. Here $\Theta(z)$ is the Heaviside step function, $\eta=\operatorname{Im}[-1 /(\varepsilon+1)]$ is the surface loss function, $\xi=\operatorname{Im}(-1 / \varepsilon)$ is the volume loss function, $\omega$ is the energy transfer, $Q$ is the parallel component of the momentum transfer, $\vec{q}$, with respect to the surface, and $Q_{ \pm}=\sqrt{[\sqrt{2 E} \pm \sqrt{2(E-\omega)}]^{2}-(\omega / v)^{2}}$. Note that we used the extended Drude dielectric function and neglected the $z$-component of $\vec{q}$ [13]. To derive surface excitations in the vacuum side, the SEPs for normally incident or escaping electrons are obtained by the integration of $\lambda_{i}^{-1}(E, z)$ over $z$ outside the solid [6]. For other crossing angles, $\alpha$, the SEPs are divided by a factor $\cos \alpha[10]$.

\section{Monte Carlo simulations and effective inelastic mean free paths}

A MC algorithm applying the depth-dependent IMFPs and SEPs has been developed previously to calculate the backscattering intensity of electrons reflected from solid surfaces $[9,10]$. In the present work, this algorithm was modified in order to apply it to a binary compound. Thus the probability of an electron being scattered by the $k$ th element in the binary compound is $\sigma_{k} /\left(\sigma_{1}+\sigma_{2}\right)$ [14], where $\sigma_{1}$ and $\sigma_{2}$ are the elastic cross sections of the component elements $k=1$, 2 . The electron elastic mean free path is calculated from $\lambda_{\mathrm{e}}=\left[N\left(\sigma_{1}+\sigma_{2}\right) / 2\right]^{-1}[15]$, where $N$ is atomic density of the compound. Note that we used a RoothaanHartree-Fock potential [16] to calculate the elastic cross sections of the individual component elements. The intensity of electrons elastically backscattered from the binary compound was calculated using the modified MC algorithm. Here a depth-independent IMFP was first applied. A relation between IMFP and intensity of backscattered electrons, i.e. the calibration curve, was then established. Subsequently, the effective IMFP was determined by a comparison of the calibration curve with the simulated intensity.

\section{Results and discussion}

Fig. 1 shows the depth-dependent inverse IMFPs calculated using Eqs. (1) and (2) for $500 \mathrm{eV}$ electrons normally incident into $(v \rightarrow s)$ or escaping from $(s \rightarrow$ v) GaAs. It is seen that the inverse IMFPs are approximately depth-independent inside the solid $(z>0)$. But they decrease rapidly with the distance from

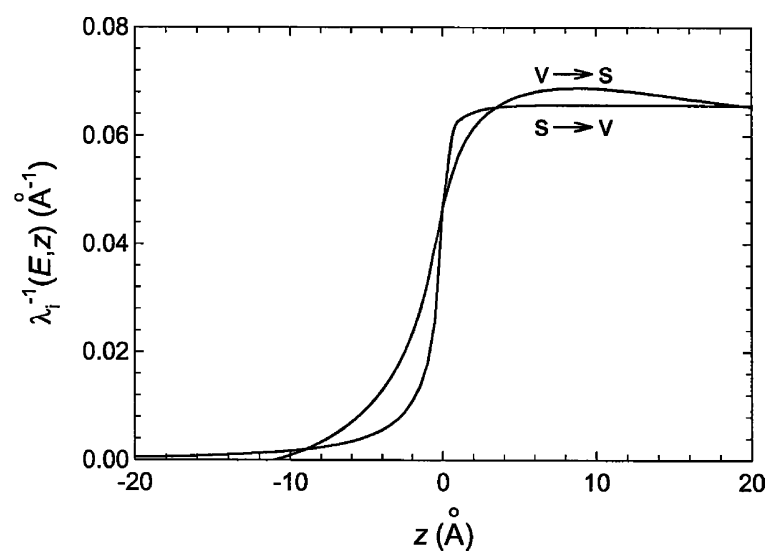

Fig. 1. A plot of the depth-dependent inverse IMFPs for $500 \mathrm{eV}$ electrons normally incident into $(v \rightarrow s)$ or escaping from $(s \rightarrow v)$ GaAs as a function of electron depth inside $(z>0)$ and outside $(z<0)$ the solid. 


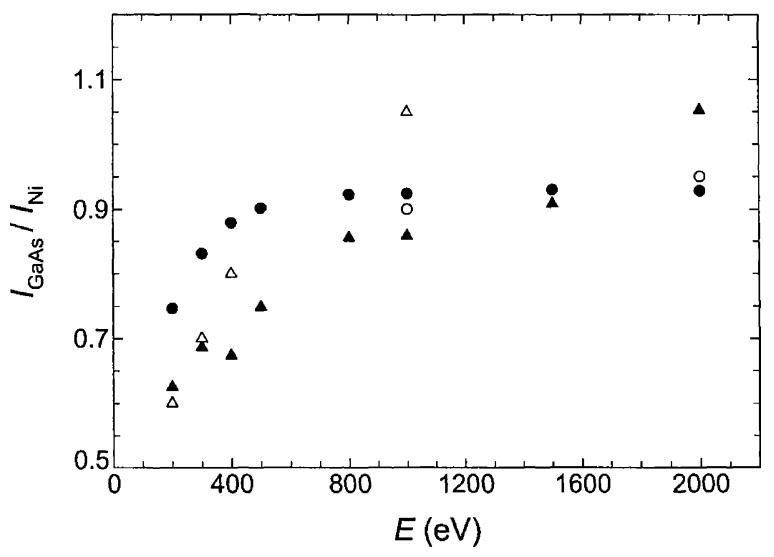

Fig. 2. The intensity ratio of electrons backscattered from GaAs to those from $\mathrm{Ni}$ for a $50^{\circ}$ incident angle and $0-90^{\circ}$ emission angles (solid circles) and for a $0^{\circ}$ (normally) incident angle and 36.3-48.3 emission angles (solid triangles). Experimental data of Krawczyk et al. (open circles) [11] and Zommer et al. (open triangles) [12] are plotted for comparison.

the surface when electrons are outside the solid $(z<0)$. Also calculated in the present work are the SEPs of electrons in GaAs. It reveals that the dependence of SEP on electron energy approximately follows $a E^{-b}$, with $a=1.4319$ and $b=0.4394$ for incident electrons and $a=1.4628$ and $b=0.4404$ for escaping electrons. The SEP for escaping electrons is slightly larger than that for incident electrons due to the electron deceleration in the former case.

Fig. 2 shows the intensity ratio of electrons backscattered from GaAs to those from $\mathrm{Ni}$ for a $50^{\circ}$ incident angle and $0-90^{\circ}$ emission angles (solid circles) and for a $0^{\circ}$ (normally) incident angle and 36.3$48.3^{\circ}$ emission angles (solid triangles). The computed elastic reflection coefficient considering surface excitations for the Ni reference material is listed in Table 1. Corresponding experimental data of the intensity ratio (open circles and triangles) [11,12] are plotted

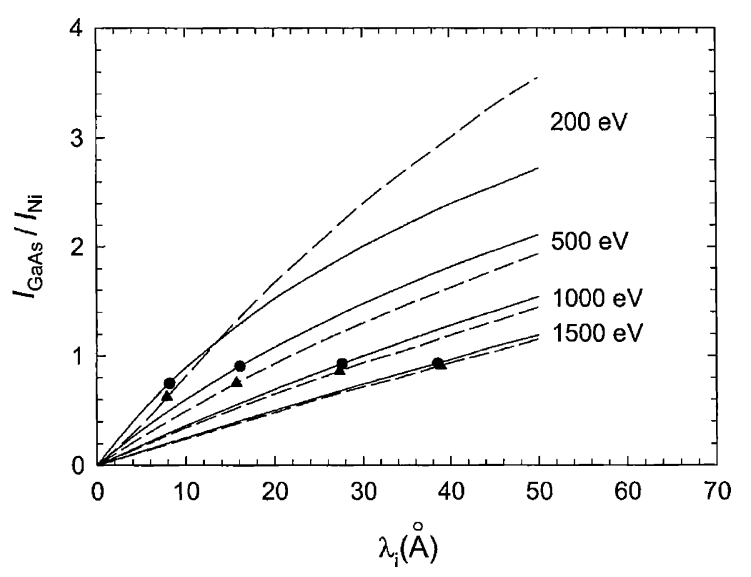

Fig. 3. The intensity ratio of electrons backscattered from GaAs to those from $\mathrm{Ni}$ calculated using the depth-independent electron IMFP (abscissa). The solid and dashed curves are, respectively, for a $50^{\circ}$ incident angle and $0-90^{\circ}$ emission angles and for a $0^{\circ}$ incident angle and $36.3-48.3^{\circ}$ emission angles. The symbols are the corresponding results obtained by MC simulations using the depth-dependent electron IMFPs and SEPs.

for comparison in Fig. 2. The discrepancy between present results and experimental data is due to the lack of information on acceptance angles used in the experiments.

Fig. 3 shows the same intensity ratio as a function of depth-independent electron IMFP, or the calibration curve, calculated using the same simulation configurations as those applied to Fig. 2. The solid and dashed curves are, respectively, for a $50^{\circ}$ incident angle and $0-90^{\circ}$ emission angles and for a $0^{\circ}$ incident angle and $36.3-48.3^{\circ}$ emission angles. The symbols are the corresponding results obtained by MC simulations using the depth-dependent electron IMFPs and SEPs. From the intersection of the calibration curve and the simulated intensity ratio, the effective IMFP may be determined. Results of the effective IMFP are plotted in Fig. 4 as a function of electron energy for a $50^{\circ}$ incident

Table 1

Values of the elastic reflection coefficient for electrons backscattered from $\mathrm{Ni}$

\begin{tabular}{llllllll}
\hline$E(\mathrm{eV})$ & & & & & \\
\hline 200 & 300 & 400 & 500 & 800 & 1000 & 1500 & 2000 \\
\hline 0.0673 & 0.0734 & 0.0726 & 0.0702 & 0.0615 & 0.0564 & 0.0462 & 0.0392 \\
$0.0079^{*}$ & $0.0100^{*}$ & $0.0128^{*}$ & $0.0132^{*}$ & $0.0116^{*}$ & $0.0105^{*}$ & $0.0074^{*}$ & $0.0057^{*}$ \\
\hline
\end{tabular}

The values are computed for a $0^{\circ}$ incident angle and 36.3-48.3 emission angles (with asterisk) and for a $50^{\circ}$ incident angle and $0-90^{\circ}$ emission angles (without asterisk). 


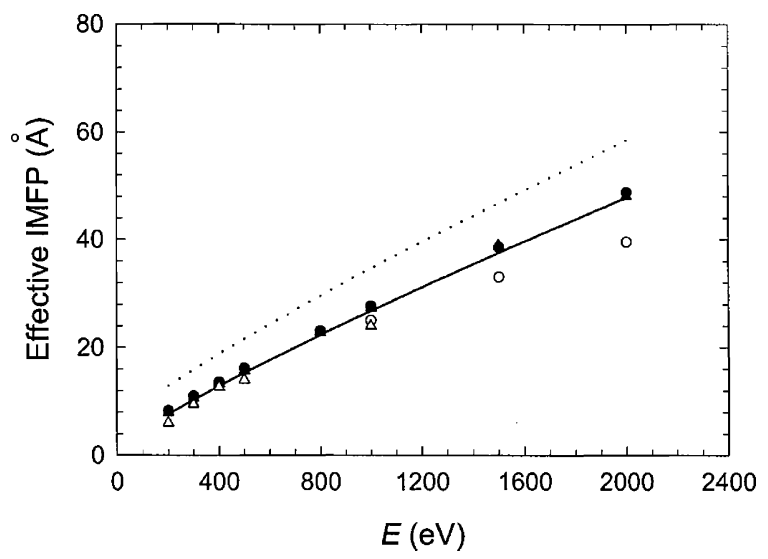

Fig. 4. The effective IMFP as a function of electron energy for a $50^{\circ}$ incident angle and $0-90^{\circ}$ emission angles (solid circles) and for a $0^{\circ}$ incident angle and $36.3-48.3^{\circ}$ emission angles (solid triangles). Also plotted are experimental data of Krawczyk et al. (open circles) [11] and Zommer et al. (open triangles) [12], theoretical results of Tanuma et al. (dotted curve) [17], and calculated IMFPs for volume excitations (solid curve).

angle and $0-90^{\circ}$ emission angles (solid circles) and for a $0^{\circ}$ incident angle and 36.3-48.3 ${ }^{\circ}$ emission angles (solid triangles). These results are compared with experimental data (open circles and triangles) [11,12], theoretical results of Tanuma et al. (dotted curve) [17], and calculated IMFPs for volume excitations (solid curve). Note that the experimental data were derived from measured intensity ratios without the consideration of surface excitations. The theoretical results of Tanuma et al. were obtained using the Lindhard dielectric function that considered no surface excitation. The effective IMFPs determined here are in good agreement with electron IMFPs for volume excitations. This indicates that a depth-independent IMFP is approximately valid due to the compensation of volume and surface excitations inside the solid $[5,6]$. Since parameters in the extended Drude dielectric function were fitted mainly for the response of valence electrons [8], the lack of inner shell responses caused the deviation between calculated effective IMFPs and experimental values at high energies.

\section{Conclusions}

The depth-dependent IMFPs and SEPs of electrons incident into and escaping from GaAs were calculated using the dielectric response theory. It revealed that inside the solid the IMFPs were roughly depth-independent due to the compensation of surface and volume excitations. Surface excitations by electrons outside the solid, characterized by the SEPs, caused a reduction in the elastic peak intensity. In the present work, both volume and surface excitations were treated in the MC simulations to calculate electron backscattered intensities. An effective IMFP was determined by a comparison of the intensity ratio from MC simulations and the calibration curve. This approach is applicable to extract the effective IMFP from the elastic intensity for a given experimental configuration and material.

Electron IMFPs and SEPs treated in the present work included the dielectric response from only valence electrons. At high electron energies, inner shell contributions become important. To include inner shell electrons, detailed analysis of all individual shell responses should be established. A similar calculation involving inner shell electrons is currently underway.

\section{References}

[1] A. Jablonski, Surf. Sci. 151 (1985) 166.

[2] S. Tougaard, I. Chorkendorff, Phys. Rev. B 35 (1987) 6570.

[3] C.J. Tung, Y.F. Chen, C.M. Kwei, T.L. Chou, Phys. Rev. B 49 (1994) 16684.

[4] Y.F. Chen, P. Su, C.M. Kwei, C.J. Tung, Phys. Rev. B 50 (1994) 17547.

[5] Y.F. Chen, C.M. Kwei, Surf. Sci. 364 (1996) 131.

[6] C.M. Kwei, C.Y. Wang, C.J. Tung, Surf. Interf. Anal. 26 (1998) 682.

[7] C.M. Kwei, Y.F. Chen, C.J. Tung, J.P. Wang, Surf. Sci. 293 (1993) 202.

[8] Y.F. Chen, Surf. Sci. 519 (2002) 115.

[9] C.M. Kwei, S.Y. Chiou, Y.C. Li, J. Appl. Phys. 85 (1999) 8247.

[10] C.M. Kwei, S.S. Tsai, C.J. Tung, Surf. Sci. 473 (2001) 50.

[11] M. Krawczyk, A. Jablonski, S. Tougaard, J. Toth, D. Varga, G. Gergely, Surf. Sci. 402 (1998) 491.

[12] L. Zommer, B. Lesiak, A. Jablonski, G. Gergely, M. Menyhard, A. Sulyok, S. Gurban, J. Elect. Spectrosc. Rel. Phenom. 87 (1998) 177.

[13] F. Yubero, S. Tougaard, Phys. Rev. B 46 (1992) 2486.

[14] P.G.T. Howell, A. Boyde, Scanning 20 (1998) 45.

[15] R. Herrmann, L. Reimer, Scanning 6 (1984) 20.

[16] E. Clementi, C. Roetti, At. Data Nucl. Data Tables 14 (1974) 177.

[17] S. Tanuma, C.J. Powell, D.R. Penn, Surf. Interf. Anal. 17 (1991) 927. 